\title{
Arts, Sciences, Religions et le surdimensionnement du cerveau humain
}

\author{
Arts, Sciences, Religions and oversized human brain
}

\author{
Jean-Pierre Gasc ${ }^{1}$ \\ ${ }^{1}$ Museum National d'Histoire Naturelle, Paris, jean-pierre.gasc@mnhn.fr
}

\begin{abstract}
RÉSUMÉ. On s'interroge sur l'universalité du fait religieux qui est socialisé sous la forme de rituels et de religions institutionnelles. L'hypertélie cérébrale caractéristique de l'être humain, conséquence de l'évolution buissonnante des grands primates, est à l'origine à la fois de sa réussite dans la vie réelle faite de luttes pour surmonter des handicaps biologiques objectifs, et d'un " travail à vide » des réseaux neuroniques pendant le sommeil, producteur d'une vie imaginaire qui échappe à la volonté et efface la frontière entre vivants et morts. Conscient de cette dualité et afin de maintenir une cohérence, l'être humain fait appel aux mythes qu'il partage dans sa vie sociale et qui participe à la cohésion des communautés. Ceci aboutit à une ritualisation qui prend les formes diversifiées des religions, souvent associée à la prise de drogues. La création artistique constitue une voie parallèle, exutoire au cerveau surdimensionné, de même que l'intuition scientifique.

ABSTRACT. One wonders about the universality of the individual religious fact that is socialized in the form of rituals and institutional religions. The cerebral hypertelism characteristic of the human being, consequence of bushy evolution of the great primates, is at the origin of both his success in real life made of struggles to overcome objective biological handicaps, and a 'vacuum work' network neurons during sleep, producer of an imaginary life that escapes the will and erase the limit between living and dead persons. Conscious for this dual aspect and to maintain coherence, the human being appeals to the myths that he shares in his social life and that contribute to the cohesion of the communities. This leads to a ritualization that takes diverse forms of religions, often joined to drugs consuming. Artistic creation is a parallel path, an outflow for the over-sized brain, as is scientific intuition.

MOTS-CLÉS. Hypertélie, rêve, hallucinogènes, chamanisme, mythologie.

KEYWORDS. Hypertelism, dream, hallucinogenic, chamanism, Mythology.
\end{abstract}

Le fait religieux peut se définir comme un ressenti à l'origine de la croyance en des entités ne relevant pas du monde tangible. Ce ressenti repose sur la recherche d'un soutien devant l'angoisse diffuse ou parfois très précise que fait naître en chaque individu le simple fait de vivre. Le fait religieux est universel, dans l'espace et dans le temps, et en raison du fait que l'être humain doit sa nature à l'existence de la structuration des individus en communauté, le ressenti individuel tend à engendrer un ensemble de croyances et de comportements qu'on appelle des religions. On a beaucoup écrit sur les religions, superstructures qui marquent l'histoire de toutes les sociétés humaines, mais on s'est très peu penché sur la source de ce phénomène intime sans lequel elles n'existeraient pas (Boyer, 2001). On peut soupçonner que ce phénomène individuel appartient aux archétypes humains, un invariant qui devient polymorphe par le biais de sa transmutation dans les divers modèles de relations sociales, qui de fait religieux se transforme en religion.

Comment relier le comportement de groupe au fait religieux, avant tout un sentiment individuel. D'où proviennent les fondements mentaux qui conduisent de façon universelle au fait religieux, une adhésion inconditionnelle à un ensemble de croyances sans fondements sur le monde réel, phénomène individuel (subjectif) qui par agrégation est à la base de la constitution d'une religion, phénomène social (collectif)? 


\section{Une production cérébrale}

Le fait religieux doit être analysé selon deux axes : l'axe historique et l'axe anthropologique. Sur le plan anthropologique, celui qui répond à des invariants de l'espèce humaine, il faut situer l'analyse au niveau des processus mentaux. Pour des raisons évolutives encore sujet de débats, la lignée des hominidés se distingue par un accroissement assez régulier et général du volume de l'encéphale correspondant à une augmentation du nombre de cellules nerveuses situées dans le cortex cérébral dont la surface est aussi considérablement accrue par son plissement, selon un processus connu dans l'évolution de nombreux mammifères. Le genre Homo atteint ainsi un indice d'encéphalisation, de rapport de la masse cérébrale sur la masse de l'organisme, qui est inégalé. Le processus s'est poursuivi pendant plus de deux millions d'années au sein de ce genre comme en témoigne les quelques fossiles connus, et une seule espèce du genre a survécu, Homo sapiens, celle à laquelle appartient l'humanité actuelle et qui est récente, moins de 200.000 années probablement.

Bien qu'il soit difficile de connaître avec précision les capacités mentales des autres espèces du genre Homo qui ont à présent disparu, on peut supposer, en raison de la proximité phylogénétique, que nous en avons conservé des traces. En particulier l'extraordinaire surabondance de neurones cérébraux devait déjà exister. Or, la capacité de ces cellules à établir des relations synaptiques multiples, à s'organiser en réseaux flexibles doués d'une grande plasticité, rend vraisemblable l'hypothèse que, au moins au sein du genre Homo, les propriétés cognitives de notre propre espèce étaient déjà esquissées. Pour avancer dans cette voie nous disposons de plusieurs faisceaux de données. Les travaux réalisés dans l'étude du comportement des autres grands primates, tout spécialement les chimpanzés et les bonobos dont la proximité génétique est désormais démontrée, nous montrent une similitude de traits qui devaient être présents chez leurs ancêtres communs, il y a plusieurs millions d'années. On sait par exemple que les grands primates reconnaissent leur propre image dans un miroir et qu'ils sont capables d'échafauder des stratégies dans les relations interindividuelles au sein du groupe. Ils sont aussi capables d'utiliser des outils pour s'aider dans une tâche liée à la prise de nourriture. Ils ne sont pas les seuls à réunir ces capacités parmi les vertébrés, certains mammifères et des oiseaux les révèlent aussi (cf revue in Andrews, 2015). Mais, ils communiquent entre eux par des vocalisations et des gestes, postures et mimiques de la face. Ils ont une certaine connaissance empirique des effets de produits naturels sur leur physiologie et sont capables de les utiliser en réponse à un besoin. Quant aux outils, ils sont fabriqués en dehors d'un besoin immédiat et révèlent une typologie qui implique leur image mentale. Ces traits comportementaux sont appris au sein de la famille qui peut se réduire au couple mère-enfant chez l'orang-outang, mais est étendue chez les gorilles et chimpanzé. Enfin, ils sont susceptibles d'être transmis de groupes à groupes et ainsi se diffuser.

Ces diverses activités nécessaires à la survie des groupes relèvent-elles des mêmes réseaux neuroniques, du même champ cognitif ? L'archéologue anglais Steven Mithen a développé une théorie qui tente une synthèse entre les connaissances acquises dans divers domaines, préhistoire, primatologie, linguistique, neurologie (1996). Il considère que l'esprit humain est doté dès la naissance de modules sous la forme de réseaux neuroniques correspondant à quelques-unes des facultés et qui seraient progressivement activés par les contacts avec le milieu naturel et culturel. Ces modules « précablés » constitueraient chez l'enfant une sorte de «couteau suisse » qui par l'expérience serait capable de construire des schémas d'action à partir de plusieurs domaines. Il suppose ainsi l'existence d'un module général qui assure l'intelligence générale, conduisant à la prise de décision par apprentissage et association, utile pour assurer la survie. A ce module seraient associés des modules plus spécialisés sur les contacts sociaux, la connaissance du monde naturel, la saisie des propriétés physiques d'objets et matériaux. Ces modules ne doivent plus être conçus comme des entités anatomiques, des «centres » dévolus à un type d'activité, mais comme des réseaux de neurones interconnectés dont l'activation par les contacts extérieurs est indispensable grâce aux entrées sensorielles. On trouve chez le neurobiologiste Edelman (2000) une idée analogue dans celle des «cartes neuronales » formant un répertoire primaire qui est transformé par une sélection entre neurones en répertoire secondaire sous l'effet des comportements. Edelman développe l'hypothèse que 
les cartes neuronales sont capables d'échanges entre elles, par un processus de «réentrée » des messages, qui peuvent ainsi se renforcer ou s'inhiber de carte en carte, les résultats étant soumis à la sélection au sein de la population de neurones par la confrontation avec les stimuli extérieurs, selon un processus que Changeux (1983 ) qualifie ainsi : «le darwinisme des synapses prend le relais du darwinisme des gènes ». Pour Mithen l'espèce actuelle, l'homme moderne, se singularise par la fluidité entre les divers modules qui, dans les espèces qui l'ont précédé et chez les grands primates, demeureraient isolés et sans échanges, ce qui interdit un enrichissement et explique une stagnation, la répétition des mêmes pratiques pendant des générations, phénomène qu'autrefois on qualifiait d'instinct.

\section{De l'importance d'un effet de groupe}

Dans le contexte de groupes plus nombreux et du passage de la cueillette d'aliments végétaux (tubercules et tiges) chez les Australopithèques à une alimentation carnée par le charognage et la chasse, les relations sociales se sont accrues au cours de l'évolution des hominidés. Ce stade correspond aussi à un accroissement significatif de la taille du cerveau, ce qui implique surtout une augmentation du nombre de neurones. Or, le passage à une alimentation carnée apporte des conditions métaboliques distinctes (Aiello and Wheeler, 1995, Leonard \& Robertson, 1996, Leonard et al.2007). D'une part, les protéines d'origine animale sont plus facilement métabolisables à l'état cru que les glucides d'origine végétale, d'autre part elles apportent deux acides gras indispensables à la croissance de l'encéphale, acide arachidonique (AA) et acide docosahexaenoïque (DHA), très peu présents dans les végétaux. Aiello remarque aussi que par un effet de balancement, le cerveau prend un volume de plus en plus important par rapport au tube digestif dont certaines fractions nécessaires à la digestion des végétaux (en particulier le gros intestin) est réduit chez les animaux carnassiers. C'est l'emploi du feu qui va permettre plus tard une utilisation plus optimisée des glucides, à partir de l'amidon de tubercules qui n'est digeste que sous forme cuite, et sans doute de diversifier le régime, donc élargir l'éventail des ressources dont la conséquence est d'assurer une croissance démographique. Le langage (un moyen de communiquer par vocalisation) se serait développé dans ce contexte en remplaçant en partie le toilettage (grooming) dans les échanges sociaux, avec l'avantage dans un groupe de maintenir un contact à distance entre plusieurs individus. Cependant, selon Mithen, le langage (peut-être sous forme de chant) n'aurait eu à l'origine de fonction d'échange que dans la sphère des relations interindividuelles au niveau des affects. En revanche il n'y aurait pas eu d'échange dans la sphère technique et de connaissance du milieu naturel où aurait agi la simple imitation. Ceci expliquerait la stagnation pendant des millénaires, qui se manifeste par la reproduction de la même technique dans la fabrication d'outils « à tout faire » (taille de bifaces) par imitation gestuelle, sans chercher à l'améliorer par un changement dans la taille ou l'emploi de nouveaux matériaux. C'est pourquoi Mithen imagine Néanderthal dans un état de conscience automatique lorsqu'il fabriquait des outils ou poursuivait une proie, quelque chose comme quand on conduit un véhicule tout en discutant, ce qu'Edelman (2000) nomme conscience primaire. A la lumière des dernières découvertes sur nos cousins Néanderthal et Denisova, il semble qu'il soit plus approprié d'envisager ce stade pour les hominidés antécédents, beaucoup plus loin dans le passé. La conscience d'ordre supérieur réside dans le fait de se reconnaître en tant qu'individu et de penser que ce qu'on ressent soi-même peut être aussi ressenti par un autre, d'où dérive sans doute l'empathie. C'est donc un fait qui a besoin d'un environnement social pour se manifester et qui existe semble-t-il chez d'autres mammifères vivant en groupe. La venue de l'homme actuel (Homo sapiens) aurait modifié rapidement les conditions, car ce serait établie une communication fluide entre les modules de l'intelligence : le secteur technique comme la connaissance de la nature seraient entrés par le langage dans le domaine social. Par l'échange verbal entre les membres du groupe, une communication des expériences individuelles crée un corpus commun de connaissances et les animaux sont chassés plus efficacement, des outils plus complexes sont élaborés pour des tâches spécialisées. En raison de la continuité assumée entre humains et non-humains dans la majorité des cultures (Descola, 2005), les animaux ont été assimilés à des humains et leur comportement devient prédictible (connaissance de leur trajet, des voies de leurs migrations); la 
chasse peut être planifiée selon le type de gibier, car les hommes se projettent en eux (d'où leur représentation sur les parois des grottes). Ce qui traduit une pleine conscience d'eux-mêmes, que l'emploi du langage facilite au sein des groupes sociaux. On ignore la cause de ce saut qualitatif dans le fonctionnement cérébral, car il s'agit manifestement d'une complexité accrue des relations entre neurones dont on ne saisit pas le déterminant. Bednarik (2011) développe l'hypothèse d'une rétroaction entre langage et complexité des relations sociales. Les découvertes le plus récentes concernant Néanderthal semblent atténuer la différence entre les deux espèces. Elle résiderait peut-être dans la différence de sensibilité à la sélection entre une adaptation stricte, morphologique et physiologique, de Néanderthal à certaines conditions climatiques, et une adaptabilité plus grande de sapiens, mais Néanderthal montre semble-t-il les mêmes rituels funéraires et perfectionnements technologiques que Cro-Magnon. Il a même produit des éléments de parure, donc une pensée symbolique. Malgré les différences morphologiques assez nettes, il est même probable que la différence ne soit pas du niveau spécifique, puisqu'on a eu la preuve moléculaire de leur hybridation en Europe. On ignore à vrai dire pourquoi seules les populations étiquetées sapiens au sein du genre Homo ont poursuivi leur expansion indifférente aux différences climatiques.

Des $900 \mathrm{~cm}^{3}$ du plus ancien Homo à $1400 \mathrm{~cm}^{3}$ en moyenne chez l'actuel, le cerveau est devenu un gros consommateur d'énergie, $20 \%$ du total de la consommation de l'oxygène pour $2 \%$ de la masse de l'organisme. L'être humain consomme par jour trois fois et demie plus d'énergie pour faire fonctionner son cerveau que les autres primates. La source de cette différence réside sans doute dans le nombre énorme de neurones groupés dans l'écorce plissée qui forment des réseaux dans lesquels circulent des signaux sous forme d'influx. Selon Leonard \& Robertson (1992) cet organe aurait détourné une partie de l'énergie nécessaire à la croissance aux dépens de la masse musculaire. En effet, contrairement à la plupart des prédateurs chez qui la masse des muscles locomoteurs représente jusqu'à $60 \%$ de la masse du corps (Calder, 1984), chez l'être humain elle atteint à peine 20 à $35 \%$. Ce cerveau a permis à de petites populations de surmonter de grands handicaps écologiques : absence d'armes naturelles contre les grands prédateurs, très faible protection contre les éléments du climat («le singe nu »), parturition problématique en raison du conflit anatomique entre la bipédie propre aux hominidés, qui implique une morphologie particulière du bassin, et la taille considérable de l'encéphale chez le nouveau-né, croissance lente avec une dépendance de la progéniture pendant de longues années, caractéristiques qui ont fait soupçonner une évolution impliquant l'hétérochronie du développement (néoténie). Or, c'est précisément les boucles de rétroaction positive entre ces attributs a priori gênants et le cerveau qui a probablement permis une sélection accélératrice.

\section{Un seul cerveau et deux vies}

Le surdimensionnement cérébral a simplement ouvert un champ de libre activité, alors qu'il apparait comme un caractère de luxe dont pourtant l'évolution montre le caractère fatal dans la plupart des lignées. Caractère résultant d'un processus que l'on nomme hypertélie, le cerveau humain est un organe qui n'est pas utilisé à plein temps dans la vie réelle, il tourne beaucoup à vide. J'entends par cette expression que pendant le sommeil le cortex cérébral ne reçoit plus d'informations qui, en provenance du monde extérieur, impliqueraient une action. En revanche, des influx proviennent aux noyaux centraux en provenance des récepteurs viscéraux et sont sans doute intégrés dans les circuits du cortex. Dickens a parfaitement traduit ceci dans «Un conte de Noël » quand il fait dire à Scrooge face au fantôme de son associé Marley « Vous n'êtes peut-être qu'un morceau de bœuf mal digéré ». Le cerveau des vertébrés est en effet un organe singulier résultant d'une propriété du neurone. La plupart des organes entrent en fonctionnement sous l'effet d'un signal déclencheur, le plus souvent chimique, ce qui leur permet de s'insérer dans une chaîne d'actions, par exemple au cours de la digestion. Ils sont donc soumis à une alternance de pause et d'activité. Même le muscle cardiaque dont le fonctionnement est continu doit être stimulé périodiquement par un signal nerveux particulier. En revanche, le système nerveux est en constante activité et de manière spontanée, assurant le contrôle et la modulation de toutes les autres activités. Le cortex qui surmonte l'ensemble du système ne cesse jamais son état actif, 
les réseaux de neurones qui le composent sont parcourus de signaux dont on peut enregistrer les effets électriques et la cessation de ces signaux signifie que l'organisme a cessé de vivre. Cette singularité est due à une propriété essentielle de tout neurone d'émettre un signal oscillant résultant d'un système en boucle, une pompe ionique, entre calcium et potassium, au niveau de la membrane cellulaire. Or, chez l'être humain actuel il y a paraît-il environ 20 milliards de neurones répartis dans les 22 décimètres carrés du cortex, organisés en réseaux qui définissent des zones fonctionnelles. Il se peut donc que le cerveau humain soit surdimensionné par rapport aux fonctions répondant aux nécessités de la vie quotidienne d'un primate de sa taille. Il y a manifestement une relation entre cet aspect quantitatif et les capacités cognitives de l'être humain. Mais on peut remarquer aussi que chez beaucoup de mammifères, en particulier les prédateurs, l'activité dans l'espace de l'environnement n'est pas permanente. Aux phases d'exploration pour la subsistance ou la reproduction succèdent des phases où l'organisme est mis au repos, hors du circuit ambiant, c'est le sommeil. Pourtant, les circuits corticaux continuent d'être parcourus par des signaux, ils sont actifs. Nous savons par notre propre expérience que cela se traduit par le sentiment d'un vécu hors du réel, le rêve.

« But then begins a journey in my head,

To work my minds, when body's work expired »

Reconnaît Shakespeare dans son sonnet 27.

Des signaux électriques particuliers sont émis par le cortex lors de cette phase du sommeil, appelée sommeil paradoxal. L'homme n'est pas le seul à rêver et c'est chez le chat que les travaux fondamentaux sur ce sujet ont été réalisés (Jouvet, 1967,1992). Cependant, les propriétés de ce ressenti sont particulièrement mises en relief chez un être qui se reconnaît comme distinct, individu, ayant conscience de lui-même et percevant comme objet ses propres sensations. Un sujet capable de se considérer comme un objet. Comment un tel être peut-il donner du sens à ce qu'il ressent comme une vie qui lui est imposée, rêves et fantasmes, mirages et hallucinations, sans doute de plus en plus complexes au cours de l'évolution et de la complexité des échanges sociaux au sein du groupe familial, clanique, sans doute très vite stimulé par la consommation de certaines substances naturelles et une vie imaginative. Ainsi, chaque être humain aurait eu très tôt à sa disposition deux vies parallèles. Une vie dure, hasardeuse, où il se confronte à la réalité des forces de la nature et aux contraintes de la vie sociale faite de conflits et de jeux d'influence. C'est là qu'il exerce ses aptitudes physiques et cognitives, qu'il peut voir, toucher les résultats de ses actions. Et une seconde vie où il a conscience que des forces insaisissables jouent de lui comme d'un objet, le plonge dans un monde où se déroule l'impossible, où les êtres morts apparaissent, partagent les actions des vivants et sont même parfois menaçants, et de cette vie il conserve la mémoire. Quelle relation entre ces deux vies ? L'une est-elle plus importante que l'autre? Où suis-je vraiment et qui suis-je? Il semble qu'il y ait deux attitudes divergentes face à ces questions. La première consiste à considérer que la vie que j'appelle imaginaire, échappant au contrôle de l'individu, révèle l'existence de forces supérieures auxquelles il faut se soumettre pour mieux affronter les obstacles de la vie réelle. Dans beaucoup de cultures subsiste ce qui a sans doute été la première tendance, dans le désir de «normaliser» la contradiction entre ces deux vies. Il s'agit de nier toute contradiction en effaçant les limites entre le monde des morts et celui des vivants, entre les images du rêve et celles de la vie quotidienne, entre les êtres humains et les éléments naturels, animaux, plantes et même les phénomènes atmosphériques. Il suffit alors de bâtir par le langage des récits justificatifs, une étiologie dit Krappe (1952), les mythes, dont la mémoire orale est maintenue par des rituels. Les mythes sont aussi une manière de placer l'histoire réelle dans le monde imaginaire, de la soustraire à la réalité, de la contrôler afin qu'elle soit conforme aux fondamentaux qui cimentent la communauté. Ainsi, c'est le même organe, le cerveau, qui d'un côté est responsable des fantasmagories inquiétantes du rêve, et de l'autre élabore une "rationalisation » par l'élaboration de mythes, afin d'échapper à l'angoisse. Il y a une « remise en ordre » sécurisante, ou comme le dit Eliade (1990) une transformation du chaos en cosmos. C'est dans ce sens que le mythe constitue une préfiguration de l'approche scientifique bien que les présupposés soient différents et, comme le 
souligne Lévi-Strauss (1962) que «la réflexion mythique apparaît comme une forme intellectuelle de bricolage » dont le propre « est d'élaborer des ensembles structurés, non pas directement avec d'autres ensembles structurés, mais en utilisant des résidus et des débris d'événements ». La transmission est orale, elle fait partie des éléments qui structurent les communautés, et ce processus oral est soutenu par une gestuelle, un ensemble de comportements qui jouent le rôle d'aide-mémoires exposés aux générations. Ce sont les rituels et les cérémonies auxquels les membres de la communauté doivent se soumettre à chaque étape de leur vie. Sinon, ils sont exclus, ce ne sont plus des « hommes » puisqu'ils ne reconnaissent pas le même « cosmos », le même monde, en un mot ils s'excluent de la culture. Pour Boyer (2001) le rituel aurait même précédé le concept de divinité. Il répond au sentiment d'urgence, quelque chose d'analogue aux TOC, une précaution obligatoire. Les rituels sont issus d'actes biologiques fondamentaux dont la répétition est une obligation physiologique, la prise de nourriture, la miction, la défécation. L'éthologie a bien documenté les diverses manifestations et comportements associés à ces actes (ritualisation) selon les espèces. L'être humain n'en est pas exclu. Il en est une autre série qui ne concerne pas seulement l'individu, mais des rapports entre individus. C'est bien sûr le cas des rapports liés à la sexualité. La ritualisation est encore plus évidente chez la plupart des vertébrés. Le plus souvent le rituel associé à un de ces actes fondamentaux emprunte des éléments comportementaux de base : piétinements, picorage, claquement des mâchoires, etc. Les espèces dont les populations sont denses et où les rapports entre individus prennent un niveau de complexité, avec de éléments de valeur (hiérarchie) propres aux organisations sociales, montrent en général un haut niveau de ritualisation (oiseaux et mammifères). Chez l'être humain, les choses se compliquent énormément par l'introduction de concepts dans les rapports de type social, depuis le niveau du noyau reproducteur élémentaire, jusqu'à celui du groupe, rassemblement d'individus conçu comme unité fonctionnelle face à «l'extérieur». Mais surtout, vient interférer cette vie imaginaire issue du fonctionnement «à vide » du cerveau. C'est alors que les rituels prennent un relief existentiel, et pas seulement de participants aux impératifs biologiques. En effet, par un phénomène de déplacement, le rituel va se substituer à l'exigence vitale du besoin physiologique auquel il est associé. Il devient superstition et par renforcement obsession compulsive, c'est-à-dire comportement automatique sans objet. C'est en cela que les rituels religieux participent au renforcement, à l'ancrage dans la nécessité du sentiment religieux individuel transformé en religion du groupe. Il existe ainsi toute une échelle de rituels, avec les différences introduite par les cultures, depuis le comportement du chat qui enterre ses excréments, jusqu'aux gestuelles répétitives dans les offices religieux associés à la transmission rigoureuse des mythes. Les TOC (Troubles Obsessionnels compulsifs) en sont les répliques pathologiques.

Par voie de conséquence, dans les cultures où l'écriture devient un auxiliaire de la mémoire, le véritable historien, celui qui raconte au plus près la réalité, sera perçu comme un blasphémateur, un dangereux hérétique, car son récit fondé sur la réalité sans cesse changeante n'"est pas conforme aux mythes et échappe au rituel répétitif. C'est pourquoi la plupart des historiens et historiographes ont participé à la consolidation du mythe, quand ils ne sont pas venus lui apporter des ornementations, pris au piège des contraintes de la communauté, c'est-à-dire de l'idéologie dominante. L'histoire a donc été jusqu'à aujourd'hui une manière d'inclure les ancêtres dans un panthéon dont le rôle est de maintenir le ciment communautaire, mais aussi de retenir les morts dans le monde des vivants. Il suffit de considérer la récurrence des appels à un «roman national » par les courants politiques conservateurs dont la rhétorique joue surtout sur le registre affectif.

La découverte, sans doute fortuite, que la consommation de certaines substances naturelles, plantes, champignons où liquides fermentés, fait surgir à la demande la vie imaginaire du rêve, est probablement très ancienne quand on considère son universalité (de Félice, 1936). Tout individu qui acquiert la maîtrise de ces usages se distingue immédiatement dans le groupe social, c'est-à-dire montre un pouvoir. Il est alors capable de franchir à volonté les limites qui séparent la vie réelle et la vie imaginaire et, en se conformant aux rituels, de servir de médiateur entre ce qui est présenté comme deux mondes, celui des vivants et celui des défunts et des ancêtres. A partir du chamanisme ou de la sorcellerie s'ouvre ainsi la voie spiritualiste qui a conduit aux religions avec toutes leurs variantes. 
Leur objectif social initial a été généralement détourné au profit des structures institutionnelles qui les soutiennent en utilisant le pouvoir que leur confèrent les éléments d'apparence surnaturelle, les « mystères ». On doit cependant remarquer que le chaman traditionnel est seul face à la communauté et doit rendre des comptes sur son efficacité. Il a d'une certaine façon obligation de résultats, ce qui n'est plus le cas lorsque se constitue une caste de religieux, une «corporation de spécialistes » (Boyer, 2001), et l'apparition de sectes, c'est-à-dire que s'institue une dépendance des autres membres de la communauté qui vont alors se comporter en «masse » (Canetti, 1966).

La seconde attitude consiste à intégrer les éléments de la vie imaginaire dans la vie réelle, et c'est la voie de l'expression esthétique qui cherche à enrichir, à rendre plus plaisante la réalité quotidienne et d'une certaine manière reprendre la main sur le vécu en le recréant. Comme le dit Francastel (1956) «L'œuvre d'art est le produit unique d'une activité qui se situe, à la fois, sur le plan des activités matérielles et des activités imaginaires d'un groupe social donné »

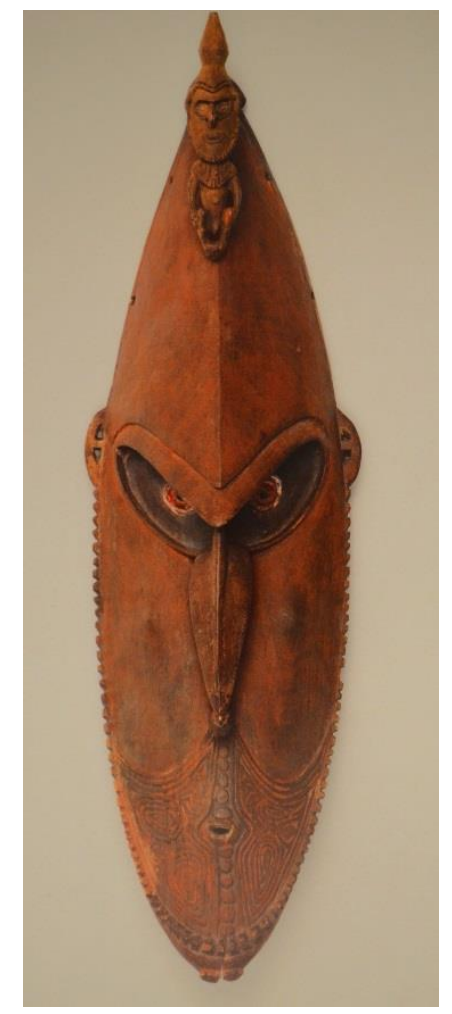

Masque Sépik, (Nouvelle Guinée-Papouasie), représentant un ancêtre. Le petit personnage au sommet est l'esprit de médiation. Weltkultur Museum, Francfort-sur-le-Main.

Ainsi le surplus d'activité cérébrale aurait été détourné par le jeu d'une rotation7 à vide dont l'individu prend conscience. Ceci fait naître le fait religieux, d'abord solution individuelle à un sentiment de discordance entre vie réelle et vie rêvée, puis conceptualisée et organisée socialement, de manière diverse selon les sociétés et leur niveau de complexité. Mais, sans doute en parallèle quand on considère l'ancienneté des grottes ornées, est née l'expression d'un monde imaginaire, sorti de l'esprit et remplaçant la réalité, représentations plastiques des activités neuronales «à vide » qui ouvrent la voie de l'abstraction. Deux dérivatifs formels donc qui se développent en parallèle, parfois convergent, souvent s'opposent, et ne suivent pas les règles de la rationalité, de la causalité. Il n'est donc pas étonnant qu'il y ait une tendance à l'anastomose entre expression du fait religieux et expression du besoin esthétique. Mythes et rituels ont trouvé un canal dans l'art, jusqu'à le parasiter et y trouver un allié, dans l'entretien d'une tradition ou pour assister le prosélytisme. L'art s'est alors trouvé soumis aux mêmes règles, aux contraintes d'une pseudo-rationalisation au service de l'institution sociale. Il a été soumis à un code de signes et de symboles au service du fait religieux, lui-même incarcéré dans des rituels puis dans des religions-institutions. Il a ainsi souvent perdu son rôle de traducteur de la vie rêvée dans le monde réel. 
Il faut néanmoins remarquer que la source de la vie imaginaire et le besoin d'exulter le trop plein de rêves ont conduit à l'expression de personnalités, à des surprises jaillissant en dehors de l'orthodoxie, insérant le fantastique et le plaisir sensoriel dans les stéréotypes mythologiques comme en témoignent les arts plastiques dans les édifices religieux. Mais il se trouve aussi parfois que la vie rêvée et l'activité de circuits en boucle débordent si fort dans la vie réelle que les exutoires que sont les mythes, les rites et l'expression esthétique ne sont plus suffisants. Alors l'individu glisse vers des positions qui le singularisent vis-à-vis du groupe social. Il est sujet à des dérèglements neuroniques dont les manifestations extrêmes, hallucinations, prostration ou logorrhée, peuvent prendre une forme en apparence organisée et structurées sur les bases préexistantes de traits sociaux ou culturels ritualisés. Des prophètes aux déments meurtriers, on rencontre ainsi des figures susceptibles d'agir puissamment sur les esprits de leur entourage et de déclencher des mouvements collectifs généralement destructeurs et suicidaires. Les mouvements messianiques conduits par des prédicateurs qui surgissent périodiquement lorsque les structures sociales se désagrègent, comme il en eu encore récemment en Amazonie et au XIXe siècle dans le Kentucky (Canetti, 1966), conduisent ainsi des foules vers leur perte assumée, car leur seule issue réside dans l'espérance de la destruction du monde, une apocalypse. L'histoire nous apprend que des personnes sans scrupule peuvent utiliser consciemment ce versant irrationnel pour mobiliser à leur profit des masses humaines, par l'intermédiaire d'êtres qui sont persuadés de posséder des dons surnaturels ou bien d'avoir été choisis par une puissance insaisissable.

\section{Du chaman aux palais royaux.}

Mais le fait religieux a aussi pour effet sur l'individu d'écarter la conscience de l'action sur le monde matériel dans le cadre de la vie réelle, pour la cantonner dans un travail à vide. A la limite cela peut conduire à un tel détachement vis-à-vis de la réalité que l'anachorète ou le mystique devient une sorte de «mort-vivant». Les penseurs au sein des religions ont compris ce danger pour le groupe social et sa cohésion. C'est pourquoi les mythes et les rites incluent une sauvegarde par une valorisation d'actions dans le monde matériel. C'est le rôle des héros ou divinités dans les polythéismes, ou bien, dans le monothéisme proche-oriental, c'est le rôle d'une exigence du Dieu visà-vis de sa créature humaine. Ainsi Gilgamesh affronte Humbaba le gardien de la forêt du Liban, il en coupe les arbres pour mériter le titre de civilisateur et roi d'Uruk, tandis que dans la Bible l'être humain devient homme et femme quand il est chassé du Paradis. Il s'agit dans les deux cas d'actions conduisant à une émancipation dangereuse, la civilisation obligée (on peut aussi penser au mythe de Prométhée qui paie cher le vol du feu aux divinités et qui se prolonge par celui de Pandore). Ainsi, le dieu d'Abraham force les hommes à affronter le monde réel et leur donne ordre de se rendre maître de la nature, dont l'exploitation devient nécessitée. La réforme protestante en fera même une garantie de salut. Mais, au cours de l'évolution des sociétés cette exploitation de la nature a pris la forme de l'exploitation de l'homme par son semblable au sein de communautés qui se stratifient par la diversité du travail née de la maîtrise de plus en plus affinée des ressources naturelles, la production de surplus et donc de la possibilité d'échanges (domestication d'animaux et agriculture au néolithique). C'est en ce sens que le fait religieux individuel, socialisé en mythes, devient alors un instrument pour justifier l'aliénation corps et esprit, des couches productrices.

Georges Dumézil (1992) a cru pouvoir reconnaître dans l'espace linguistique indo-européen la généralité d'une stratification des sociétés en trois ordres, l'ordre guerrier, l'ordre des prêtres et celui des producteurs de biens. La réalité historique révèle bien une tendance de ce genre mais aussi une plus grande complexité selon les cas, les temps et les cultures. La première strate à se distinguer est sans doute celles des chamans, devins et prêtres, les reste de la communauté se distinguant essentiellement par une différenciation des activités selon le sexe et l'âge. La strate des guerriers n'a dû se constituer de façon permanente (et non pas conjoncturelle par le groupement de chasseurs à l'occasion d'une opération de raid) lorsque la domestication de plantes et d'animaux a permis l'accumulation de réserves qui ont attiré les convoitises donc nécessité une protection. Le pouvoir, c'est-à-dire le lieu de prise de décisions qui engagent l'avenir de la communauté, a oscillé entre les prêtres et les guerriers, 
les premiers possédant des forces occultes, les seconds disposant des forces matérielles des armes. Le degré de perméabilité des esprits guerriers aux superstitions (et supercheries) et déguisements rituels a joué un rôle important dans cette oscillation du pouvoir. La ségrégation de ces deux strates ayant un pouvoir apparaît par l'existence de quartiers distincts dans les premières villes ce qui reflète sans doute une précaution politique. En même temps l'existence de guerriers permanents permettait à la strate religieuse de maintenir leur pouvoir sur les producteurs en toute circonstance, par exemple pendant les disettes et les épidémies devant lesquelles l'impuissance des forces occultes devenait source de révoltes. Dans le monde proche-oriental et méditerranéen, à partir du $3^{\mathrm{e}}$ millénaire, c'est une hiérarchie fondée sur un chef de guerre qui prédomine. Il cumule la fonction religieuse en tant qu'ordonnateur des rites. Le cuivre est devenu la matière première, et remplace la pierre (silex ou obsidienne), le char tiré par un cheval, invention venant probablement des steppes asiatiques, est l'apanage des guerriers. La maîtrise sans doute ancienne de la navigation n'est plus un moyen de migrer mais un outil de communication et de conquête. Mais l'essentiel est économique : établissement de comptoirs dans des pays lointains, échanges de productions et accumulation de richesses caractérisent les sociétés de ces époques, qu'elles soient insulaires (par exemple à Chypre, et en Crète) ou continentales. Il en découle l'émergence d'un nouveau groupe social, celui qui maîtrise l'écriture et jouent le rôle de comptables autant que de chroniqueurs, ce qui d'ailleurs nous a permis de connaitre certaines formes de cette organisation sociale. Le Palais dont on trouve le prototype en Crète devient une forme d'état organisant des activités très diverses, productions agricoles et d'élevage, artisanat utilitaire et spécialisé. Les cultes sont célébrés dans des édifices particuliers, des «temples» présents dans les villes de Mésopotamie et d'Anatolie, sous la responsabilité d'une classe spécialisée. Mais ce n'est pas toujours le cas. En Crète par exemple, les cultes, très liés aux rythmes agraires, sont célébrés dans des grottes, ancien type d'habitation au néolithique, ou bien au sommet de montagnes, ce qui dénote une absence de véritable ségrégation d'une classe religieuse associée à des constructions. En revanche, il semble que dans cette île les femmes jouaient le rôle d'officiants spécialisés pour les célébrations, tandis que nombre de divinités étaient féminines. Nature, jeunesse, agilité et négation de la mort par la résurrection périodique, semblent constituer les lignes fondamentales des rites minoens qui paraissent à cet égard bien particuliers.

En Chine les conditions sont très différentes car il n'y a jamais eu de véritables religions institutionnelles, avec une classe de prêtres participant au pouvoir. Ce pouvoir s'est organisé autour d'un système matériel d'administration des choses et lorsqu'un empereur, hissé à ce niveau par la force des armes, reçoit une qualification surnaturelle c'est en tant d'intermédiaire entre le système d'administration des choses et un système symétrique d'administration du cosmos, du ciel (Cheng, 1997). Quant aux pratiques religieuses elles demeurent au niveau du chamanisme et de son rôle divinatoire, celui de s'assurer de l'avenir. Ces pratiques sont exercées par des individus et sont monnayées. Leur but est de s'assurer une protection contre les forces de la vie imaginaire et l'intrusion des morts, fruits du surplus de l'activité cérébrale. Ceci explique qu'en Chine et dans les empires mongols, les diverses formes de religions apparues au Proche-Orient et en Arabie, et diffusées par les routes commerciales de l'Asie centrale, ne sont pas entrées en concurrence avec une religion officielle, c'est-à-dire associée au pouvoir, et ont souvent coexisté au sein de l'Empire sans être inquiétées, tant qu'elles n'ont pas cherché à influencer le pouvoir. Ce qu'on appelle religions, taoïsme, confucianisme et le bouddhisme importé de l'Inde, sont essentiellement des philosophies donnant à l'Empire les fondements logiques et existentiels aux règles d'administration des choses et des hommes. Il est vrai que ces ensembles, agnostiques à l'origine, ont donné des formes syncrétiques en incorporant des pratiques anciennes issues sans doute du chamanisme, prenant alors des formes religieuses avec souvent une multitude de divinités populaires et un corps d'officiants, mais sans recherche du pouvoir politique. Il n'y a pas eu en Chine de guerres pour des motifs religieux. 


\section{Références}

Aiello L.C., Wheeler P. 1995. The expensive-Tissue Hypothesis: The Brain and the Digestive System in Human and Primate Evolution. Current Anthropology, 36,2:199-221.

Boyer P. 2001. Et l'homme créa les dieux. Comment expliquer la religion, Paris, Robert Laffont.

Calder W.A. 1984. Size, Function and Life History. Cambridge, Ma, Harvard University Press.

Canetti E. 1966. Masse et puissance. Paris, Gallimard.

Changeux J-P 1983. L'homme neuronal. Paris,Fayard.

Cheng A. 1997. Histoire de la pensée chinoise. Paris, Editions du Seuil.

Descola P. 2005. Par-delà nature et culture. Gallimard, Partis ;

Dumézil, G., 1992. Mythes et Dieux des Indo-européens, Paris, Flammarion.

Edelman G.M. 2000. Biologie de la conscience, Paris, Odile Jacob.

Eliade, M.,Couliano,J.P. 1990. Dictionnaire des religions. Paris, Plon.

Felice P. de. 1936. Poisons sacrés Ivresses divines : Essai sur quelques formes inférieures de la mystique. Paris, Albin Michel.

Francastel,F. 1956. Art et technique. Paris, Editions de Minuit.

Jouvet F. 1967. The States of Sleep. Scientific American,216 (2) :62-68.

Id 1992. Le Sommeil et les rêves. Paris, Odile Jacob.

Kant E. 1784. Beautwortung der Fraze : Was ist Aufklärung ? Berlinische Monatschrift.

Krappe, A.H. 1952. La genèse des mythes. Paris Payot.

Leonard W. R. \& Robertson, M. I., 1992. Nutritional Requirements and Human Evolution: A Bioenergetics Model. American Journal of Human Biology. 4:179-195.

Leonard W. R. \& Robertson, M. I., and J. Snodgrass. 2007 Energetics and the Evolution of Brain Size in Early Homo. In, Guts and Brains, An Integrative Approach to the Hominid Record, Wil Roebroeks (ed.) Leiden University Press: 2943.

Lévi-Strauss C. 1962. La pensée sauvage. Paris, Plon.

Mithen S. 1996. The Prehistory of Mind: The Cognitive Origins of Art and Science. London,

Mithen S. 2005. The Singing Neanderthal. The Origins of Music, Language, Mind, and Body. Cambridge, Ma, Harvard University Press. 